\title{
Illusory patterns are fishy for fish, too
}

\section{Christian Agrillo *, Maria Elena Miletto Petrazzini and Marco Dadda}

Department of General Psychology, University of Padova, Padova, Italy

*Correspondence: christian.agrillo@unipd.it

\section{Edited by:}

Davide Zoccolan, International School for Advanced Studies, Italy

Reviewed by:

Davide Zoccolan, International School for Advanced Studies, Italy

Vera Schluessel, Rheinische Friedrich-Wilhelm Universität Bonn, Germany

Keywords: visual illusion, comparative perception, fish, illusory pattern, animal models

It has been widely recognized that size, shape, and distance perception are not the mere translation of images in the eyes, as retinal images are inherently ambiguous. Some form of knowledge and/or assumptions by unconscious inductive inference seems to be necessary (Gregory, 1997). With respect to this topic, visual illusions are a valuable tool for understanding the neuro-cognitive systems underlying visual perception by indirectly revealing the hidden constraints of the perceptual system in a way that normal perception cannot. In humans, such constraints have been often summarized as the so-called "Gestalt principles," which can be briefly described by the motto "the whole is greater than the sum of its parts" (Wertheimer, 1938). Almost a century of experimental investigation on visual illusions has broadened our comprehension of the perceptual mechanisms that enable us to perceive figures and forms instead of just a collection of lines and curves. Such mechanisms are highly adaptive, as they allow for a quick and stable picture of the environment, enabling an appropriate motor response in every context (Ikin and Turner, 1972).

Given their high ecological value, there is little reason to believe that selective pressures to develop a visual system that is able to segregate objects from the background have acted only on hominids. Indeed, over the last decade, research has demonstrated that both apes and monkeys are deceived by illusory patterns. For instance, baboons perceive the Zöllner illusion (Benhar and Samuel, 1982), capuchin monkeys perceive the Müller-Lyer illusion (Suganuma et al., 2007), and rhesus monkeys perceive numerosity illusion (Beran, 2006; Beran and Parrish, 2013), thus showing that the organization of visual information is similar between human and non-human primates.
Despite the existence of a large number of studies, it is still unclear to what extent previous experience plays a role in how the brain/mind interprets and reconstructs physical reality (Hebb, 1949; Bod, 2002; Quinn and Bhatt, 2006). For practical and ethical reasons, it is very difficult to manipulate experiences during developmental periods in human and non-human primates. Furthermore, as primates lack independence at birth, different procedures are used for studying newborns, juveniles, or adults, presenting one of the major drawbacks when studying the development of visual perception in primates, i.e., the difficulty of devising experimental paradigms applicable to different ages (Bisazza et al., 2010). The recent discovery that even relatively simple organisms like fish, whose divergence seemingly occurred approximately 450 million years ago (Kumar and Hedges, 1998), also perceive visual illusions, as humans do, paves the way for the use of new animal models to investigate the relative contribution of genes and experience.

Redtail splitfin, for instance, was shown to be able to perceive illusory contours (Sovrano and Bisazza, 2009). Fish were required to discriminate between a square or a triangle and the corresponding background. After reaching a learning criterion, subjects performed test trials in the presence of two stimuli: one consisted of a subjective figure (triangle or square) induced by interruption or spatial phaseshift of diagonal lines; the other consisted of a series of diagonal lines only. In a subsequent test, two figures were presented: one in which pacmen were positioned in order to reproduce the Kanizsa triangle or square, and one in which the same pacmen were scrambled in different positions so as to prevent an impression of a subjective figure. Discrimination of orientation, rather than discrimination of shape, was also tested in a second experiment. Subjects were initially trained to discriminate between a vertical and a horizontal line with real physical contours. In test trials vertically and horizontally oriented illusory lines were presented, created either through interruption or spatial phase-shift of diagonal lines (see Table 1). Redtail splitfin were found to perceive illusory contours in both experiments.

Wyzisk and Neumeyer (2007) successfully trained goldfish to discriminate between triangles and squares. After reaching the learning criterion, the authors presented a Kanizsa triangle and a Kanizsa square, and found that goldfish were able to discriminate between the two patterns based on the illusory contours. Goldfish showed high orientation sensitivity with respect to the pacmen generating the illusory patterns. Interestingly, if black lines were over-imposed on a Kanizsa triangle or square, the illusory perception was disrupted, as has also been reported in humans, suggesting the existence of an end-stopped property similar to the neurons in V2 found in monkeys (von der Heydt, 2004).

Data collected on redtail splitfin and goldfish are particularly interesting as the two species are only distantly related. According to recent estimates, the Ostariophysi, the group to which redtail splitfin belong, and the Acanthopterygii, the group to which goldfish belong, diverged more than 250 million years ago (Steinke et al., 2006). The fact that even distantly related species perceive illusory contours suggests the existence of orientation-selective neurons-responding to edges, lines, or bars of high contrast-in a wide range of teleost fish. Also, more recent evidence further suggests similar perceptual 
Table 1 | Summary of static illusory patterns investigated in teleost fish (chronological order).

\begin{tabular}{|c|c|c|c|}
\hline Authors & Species & Type of illusion & $\begin{array}{l}\text { Schematic representation } \\
\text { of stimuli }\end{array}$ \\
\hline Wyzisk and Neumeyer, 2007 & Carassius auratus & Illusory contours & \\
\hline Sovrano and Bisazza, 2008 & Xenotoca eiseni & Amodal completion & \\
\hline Sovrano and Bisazza, 2009 & Xenotoca eiseni & Illusory contours & \\
\hline Darmaillacq et al., 2011 & Variola louti and Scarus niger & Amodal completion & \\
\hline
\end{tabular}

mechanisms between fish and primates: reef fish tested in their natural environment exhibited amodal completion, as they tried to attack their own mirror image even when they could see a fragmented image of themselves (Darmaillacq et al., 2011). It is interesting to note that fish did not attack their imagine when they could see only a portion of the body in a single square, thus showing that their aggressive behavior was not simply triggered by some specific body features, such as color. Amodal completion was also reported in another fish species, the redtail splitfin (Sovrano and Bisazza, 2008).

These studies have theoretical implications in the debate surrounding human visual perception. It has been suggested that a single unit-formation process may underlie modal (the perception of both real and subjective contours) and amodal completion, as completion processes would depend on a common underlying mechanism connecting edges across gaps (Kellman et al., 1998; Palmer, 1999). Fish species reported in the literature (Table 1) showed a successful perception of both modal and amodal completion. This finding indirectly aligns with the idea of a single mechanism for the two processes. Nonetheless, we believe that future research on newborn and juvenile fish will provide even more useful insights, especially in the debate surrounding the developmental trajectories of Gestalt principles. Due to their relatively short lifespan and independence at birth, fish represent an excellent experimental model for studying the development of perception and cognition. Indeed, recent studies have already adopted fish to study the ontogeny and the developmental trajectories of perceptual and cognitive systems (Bisazza et al., 2010; Miletto Petrazzini et al., 2013). Given that adult fish vision seems to be based on Gestalt principles, the development of such principles may be now investigated using newborn/juvenile fish as a model.

A validated method exists to study cognition and perception in newborn fish (Miletto Petrazzini et al., 2012). This method involves introducing two stimuli (i.e., two different geometric figures) at the opposite ends of the tank and delivering food near the discriminative stimulus. Discrimination is inferred from the portion of time spent near the trained stimulus during final probe trials. The method has been shown to be very rapid (only 12 reinforced trials) and successful in discrimination tasks (i.e., circle vs. triangle), thus making it a good candidate for investigating the ontogeny of Gestalt principles in rapidly growing species, such as fish. Based on previous literature, the focus should be given initially to illusory patterns called "Fictions"-including illusory contours-in the classification advanced by Gregory (1997). First, it would be interesting to see if/which Gestalt principles are inherent; if not, it would be challenging to study their developmental trajectory and the influence of maturation and experience.

The use of zebrafish, one of the main model organisms for neurobiology studies of vision and neurodevelopmental genetics, is especially welcome, given the possibility to extend the investigation on illusory perception with genetic and neuroanatomic aspects. The anatomical, physiological, and genetic components of the zebrafish visual system have been widely investigated in both larval and adult individuals (e.g., Bilotta and Saszik, 2001). Several studies indicate that zebrafish are capable of high-level motion processing. In particular, two visually guided behaviors received great attention in the literature: the optokinetic response (OKR) and the optomotor response (OMR). The OKR is a consistent behavior in which moving objects across the visual field evoke stereotyped eye movements (Neuhauss, 2003; Huang and Neuhauss, 2008). These eye movements consist of two distinct components: a smooth pursuit movement and a fast saccade which resets the eyes once the object has left the visual field (Portugues and Engert, 2009). A small hindbrain area in rhombomere 5 has been found to be necessary for this response to occur properly (Schoonheim et al., 2010). Neuhauss et al. (1999) found that zebrafish mutant belladonna ( $b e l$ ) often displays an OKR opposite to the direction of movement of the objects. Interestingly, Huang et al. (2009) found that a subset of the same mutants also display atypical circular swimming patterns ("looping") as a result of illusionary self-motion perception. On the other hand, the OMR occurs when a whole-field moving stimulus is presented and the fish turn and swim according to the perceived motion direction (Neuhauss et al., 1999; Portugues and Engert, 2009). 
Mutants with visual defects-such as the lakritz(lak) mutant, which lacks a large subset of retinal ganglion cells-fail at the OMR test (Baier, 2000).

In humans, both OKR and OMR have been hypothesized to be involved in different visual illusions (Schor et al., 1984; Riecke et al., 2009). In this sense, the use of mutant zebrafish with opposite OKR, or lacking OMR, will play a key role in verifying the influence of both neural mechanisms in the perception of illusory patterns in a way that is not possible with primates.

Small brains are likely to provide important insights with respect to the ancient philosophical question of how the visual system builds our reality.

\section{ACKNOWLEDGMENTS}

The authors would like to thank Alexandra Protopopova and the reviewers for their useful comments.

\section{REFERENCES}

Baier, H. (2000). Zebrafish on the move: towards a behavior-genetic analysis of vertebrate vision. Curr. Opin. Neurobiol. 10, 451-455. doi: 10.1016/S0959-4388(00)00116-1

Benhar, E., and Samuel, D. (1982). Visual illusions in the baboon (Papio anibis). Anim. Learn. Behav. 10, 115-118. doi: 10.3758/BF03212056

Beran, M. J. (2006). Quantity perception by adult humans (Homo sapiens), chimpanzees (Pan troglodytes), and rhesus macaques (Macaca mulatta) as a function of stimulus organization. Int. J. Comp. Psych. 19, 386-397.

Beran, M. J., and Parrish, A. E. (2013). Visual nesting of stimuli affects rhesus monkeys' (Macaca mulatta) quantity judgments in a bisection task. Atten. Percept. Psychophys. 75, 1243-1251. doi: 10.3758/s13414-013-0474-5

Bilotta, J., and Saszik, S. (2001). The zebrafish as a model visual system. Int. J. Dev. Neurosci. 19, 621-629. doi: 10.1016/S0736-5748(01)00050-8

Bisazza, A., Piffer, L., Serena, G., and Agrillo, C. (2010). Ontogeny of numerical abilities in fish. PLoS ONE 5:e15516. doi: 10.1371/journal.pone.0015516

Bod, R. (2002). Memory-based models of melodic analysis: challenging the gestalt principles. J. N. Mus. Res. 31, 27-37. doi: 10.1076/jnmr.31.1.27.8106

Darmaillacq, A. S., Dickel, L., Rahmani, N., and Shashar, N. (2011). Do reef fish, Variola louti and Scarus niger, perform amodal completion? evidence from a field study. J. Comp. Psychol. 125, 273-277. doi: 10.1037/a0024295

Gregory, R. (1997). Visual illusions classified. Trends Cogn. Sci. 1, 190-194. doi: 10.1016/S1364-6613(97)01060-7

Hebb, D. O. (1949). The Organization of Behavior. New York, NY: John Wiley.

Huang, Y. Y., and Neuhauss, S. C. (2008). The optokinetic response in zebrafish and its applications. Front. Biosci. 13, 1899-1916.

Huang, Y. Y., Tschopp, M., and Neuhauss, S. C. (2009). Illusionary self-motion perception in zebrafish. PLoS ONE 4:e6550. doi: 10.1371/journal.pone.0006550

Ikin, M., and Turner, J. R. G. (1972). Experiments on mimicry: gestalt perception and the evolution of genetic linkage. Nature 239, 525-527. doi: 10.1038/239525b0

Kellman, P. J., Yin, C., and Shipley, T. F. (1998). A common mechanism for illusory and occluded object completion. J. Exp. Psych. Hum. Percept. Perform. 24, 859-869. doi: 10.1037/0096-1523. 24.3.859

Kumar, S., and Hedges, S. B. (1998). A molecular timescale for vertebrate evolution. Nature 392, 917-920. doi: 10.1038/31927

Miletto Petrazzini, M. E., Agrillo, C., Piffer, L., and Bisazza, A. (2013). Ontogeny of the capacity to compare discrete quantities in fish. Dev. Psychobiol. doi: 10.1002/dev.21122. [Epub ahead of print].

Miletto Petrazzini, M. E., Agrillo, C., Piffer, L., Dadda, M., and Bisazza, A. (2012). Development and application of a new method to investigate cognition in newborn guppies. Behav. Brain Res. 233, 443-449. doi: 10.1016/j.bbr.2012.05.044

Neuhauss, S. C., Biehlmaier, O., Seeliger, M. W., Das, T., Kohler, K., Harris, W. A., et al. (1999). Genetic disorders of vision revealed by a behavioral screen of 400 essential loci in zebrafish. J. Neurosci. 19, 8603-8615.

Neuhauss, S. C. (2003). Behavioral genetic approaches to visual system development and function in zebrafish. Dev. Neurobiol. 54, 148-160. doi: 10.1002/neu.10165

Palmer, S. E. (1999). Organizing objects and scenes, in "Vision Science: Photons to Phenomenology," ed S E Palmer (Cambridge, MA: MIT Press), 254-310.

Portugues, R., and Engert, F. (2009). The neural basis of visual behaviors in the larval zebrafish. Curr. Opin. Neurobiol. 19, 644-647.

Quinn, P. C., and Bhatt, R. S. (2006). Are some Gestalt principles deployed more readily than others during early development? The case of lightness versus form similarity. J. Exp. Psych. Hum. Percept. Perform. 32, 1221-1230. doi: 10.1037/0096-1523.32.5.1221

Riecke, B. E., Valjamae, A., and Schulte-Pelkum, J. (2009). Moving sounds enhance the visually-induced self-motion illusion (circular vection) in virtual reality. ACM Trans. Appl. Percept. 6, 7-27. doi: 10.1145/1498700. 1498701

Schoonheim, P. J., Arrenberg, A. B., Del Bene, F., and Baier, H. (2010). Optogenetic localization and genetic perturbation of saccade-generating neurons in zebrafish. J. Neurosci. 30, 7111-7120. doi: 10.1523/JNEUROSCI.5193-09.2010

Schor, C. M., Lakshminarayanan, V., and Narayan, V. (1984). Optokinetic and vection responses to apparent motion in man. Vis. Res. 24, 1181-1187. doi: 10.1016/0042-6989(84)90173-1

Sovrano, V. A., and Bisazza, A. (2008). Recognition of partly occluded objects by fish. Anim. Cogn. 11, 161-166. doi: 10.1007/s10071-007-0100-9

Sovrano, V. A., and Bisazza, A. (2009). Perception of subjective contours in fish. Perception 38, 579-590. doi: 10.1068/p6121

Steinke, D., Salzburger, W., and Meyer, A. (2006). Novel relationships among ten fish model species revealed based on a phylogenomic analysis using ESTs. J. Mol. Evol. 62, 772-784. doi: 10.1007/s00239-005-0170-8

Suganuma, E., Pessoa, V., Mongefuentes, B., Castro, B., and Tavares, M. (2007). Perception of the Müller-Lyer illusion in capuchin monkeys (Cebus apella). Behav. Brain Res. 182, 67-72. doi: 10.1016/j.bbr.2007.05.014

von der Heydt, R. (2004). "Image parsing mechanisms of the visual cortex," in The Visual Neurosciences, eds L. M. Chalupa and J. S. Werner (Cambridge, MA: MIT Press), 1139-1150.

Wertheimer, M. (1938). "Laws of organization in perceptual forms," in A Sourcebook of Gestalt Psychology, ed W. B. Ellis (Harcourt: Brace and Company), 71-88.

Wyzisk, K., and Neumeyer, C. (2007). Perception of illusory surfaces and contours in goldfish. Vis. Neurosci. 24, 291-298. doi: $10.1017 /$ S095252380707023X

Received: 02 July 2013; accepted: 07 August 2013; published online: 28 August 2013.

Citation: Agrillo C, Miletto Petrazzini ME and Dadda $M$ (2013) Illusory patterns are fishy for fish, too. Front. Neural Circuits 7:137. doi: 10.3389/fncir.2013.00137

This article was submitted to the journal Frontiers in Neural Circuits.

Copyright (c) 2013 Agrillo, Miletto Petrazzini and Dadda. This is an open-access article distributed under the terms of the Creative Commons Attribution License (CC BY). The use, distribution or reproduction in other forums is permitted, provided the original author(s) or licensor are credited and that the original publication in this journal is cited, in accordance with accepted academic practice. No use, distribution or reproduction is permitted which does not comply with these terms. 